\title{
VIEWPOINT
}

\section{NICE: guessing is not guidance}

\section{P A Poole-Wilson}

A preliminary assessment of the extent to which the National Institute for Clinical Excellence (NICE) has achieved its goals within cardiovascular medicine is presented. NICE is a government sponsored agency operating in the UK which attempts to assess the value of medical therapies, devices, and surgery for a wide range of medical and surgical problems. Its recommendations determine whether a particular therapy can be adopted in the National Health Service.

T he creation of the National Institute for Clinical Excellence (NICE) was a timely initiative, setting up a body which would make recommendations to those working in the National Health Service and elsewhere on the effective use of current treatments. Treatments were to include drugs, devices, and interventional procedures including surgery. Until now the use of drugs or devices has been largely controlled by the Committee for the Safety of Medicines (CSM), the Medicines Control Agency (MCA), and the Medical Devices Agency (MDA). Pharmaceutical companies submitted evidence, usually from clinical trials, to these bodies whose role was to assess the benefit to patients before approving a drug and granting a licence. Device companies followed a somewhat similar procedure. The introduction of new surgical techniques or innovative operations was not overseen and was in effect managed by the medical profession itself. An objective of NICE was to move away from the simple demonstration of efficacy (effects in clinical trials) towards effectiveness (effects in clinical practice within the community) and to efficiency (cost effectiveness). That was and is a laudable objective.

NICE (www.nice.org.uk) criticises published guidelines arguing that they vary in quality and can be opinionated, biased, and contradictory and take little account of effectiveness or efficiency. NICE states: "NICE guidelines are based on the best available research evidence and expert professional advice. They take into account both clinical effectiveness and cost effectiveness, and must be practical and affordable." On the back of the cover page of documents entitled "Technology appraisal guidance" it is stated: "This guidance represents the view of the Institute's Appraisal Committee .... Health professionals are expected to take it fully into account when exercising their clinical judgement ... This guidance does not however override the individual responsibility of health professionals to make appropriate decisions in the circumstances of the individual patient, in consultation with the patient and/or guardian or carer."
NICE has now issued 16 technology appraisal guidance documents (15 numbered plus the first on Zanamivir in October 1999). Three of these documents are relevant to cardiovascular medicine, namely those on stents (document 4), implantable cardioverter-defibrillators (ICDs) (document 11), and glycoprotein IIb/IIIa inhibitors (document 12). A preliminary assessment of the extent to which NICE has achieved its goals within cardiovascular medicine and kept to its stated criteria is now timely.

\section{TECHNOLOGY APPRAISAL GUIDANCE 4: GUIDANCE ON CORONARY ARTERY STENTS IN THE TREATMENT OF ISCHAEMIC HEART DISEASE}

NICE advocates the use of stents routinely where percutaneous coronary intervention is recommended for angina or acute myocardial infarction, and states: "Where it is considered clinically appropriate to undertake either PCI (percutaneous coronary intervention) or coronary artery bypass grafting (CABG), the availability of stents should push the balance of clinical decisionmaking towards PCI". These proposals are not in line with current practice. Four features of this document are notable.

Those who were asked for opinions included the manufacturers of stents, specialist societies, and two physicians. No surgeon appears to have been asked to participate in the writing of this document, nor does it appear that any of the surgical societies were consulted. That is surprising in view of the statements made in relation to the choice of stent or surgery.

In the presence of complex lesions current practice is to insert a stent. In large trials patients with stents have been shown to have a more favourable outcome compared to those with standard PTCA (percutaneous transluminal coronary angioplasty), but it may be that selected patients with simple lesions and an optimal result from PTCA do as well as those in whom a stent has been inserted. The long term outcome of stent insertion remains unknown.

A more difficult decision for the cardiologist is the choice between insertion of a stent or proceeding to CABG on or off bypass. That issue is subject to current trials.

One of these trials, ARTS (arterial revascularization therapy study), is not completed but preliminary results have been published. The second trial, SoS (stent or surgery), will probably be reporting in March 2001. What is known at present would suggest that there is a marginal benefit in favour of surgery compared to a stent in terms of outcome assessed as a composite of several end points. The conclusion from this document appears to be an informed guess as to the 
outcome of those as yet unpublished studies from groups not representing the alternative procedure namely surgery.

The fourth issue is why this topic was chosen as the first in the arena of cardiovascular medicine. It is a relatively simple topic which does not greatly trouble physicians or surgeons in their decision making. Far more difficult are the questions of when patients with coronary artery disease should undergo intervention and which type of intervention is the most appropriate. Medical treatment has advanced substantially. Numerous catheter based treatments are available. Likewise surgical procedures have improved and changed; information on cardiac surgery is based on trials undertaken many years ago. In Britain fewer interventional procedures, be they PCI or surgery, are undertaken than in the rest of Europe. That is the major issue facing cardiologists, not the nature of the procedure to be undertaken during PCI.

\section{TECHNOLOGY APPRAISAL GUIDANCE 11: GUIDANCE ON THE USE OF IMPLANTABLE CARDIOVERTER-DEFIBRILLATORS FOR ARRHYTHMIAS}

Implantable cardioverter-defibrillators (ICDs) are used less in the UK than in the rest of Europe. The guidance arising from NICE will undoubtedly increase the use of these devices and increase costs contrary to what might have been the worst expectations of the cardiological community. To that extent the guidelines must be welcomed.

Consultation on this document was somewhat limited. Since the link between the use of these devices and the management of heart failure is so crucial wider consultation would have been appropriate. It is arguable that a greater benefit would emerge from the implementation of preventive strategies for coronary heart disease and from the greater use of $\beta$ blockers in heart failure. ICDs would be largely reserved for a selected group of patients, particularly those with cardiac arrest, sustained ventricular tachycardia, ventricular fibrillation or inherited susceptibility to arrhythmias, all in the absence of coronary heart disease. Such hypotheses need testing.

Section 2.2 of the document leads the reader to equate sudden cardiac death with an arrhythmic death. In one sense all sudden deaths must be arrhythmic, but there is increasing evidence that many deaths in this category are instigated by undiagnosed ischaemic events or bradycardia, particularly in the context of heart failure. In discussing sudden cardiac death a distinction is necessary between those with or without coronary heart disease and between those with or without heart failure.

The main current recommendations are presumably based on the outcomes of recent trials such as AVID, MADIT, CIDS, CASH, MUSTT, and CABG Patch. The most controversial part of this guideline is section 1.1.2 where "primary prevention" includes patients with heart failure. A simple reading would suggest that patients with heart failure (ejection fraction $<35 \%$ and NYHA functional class III), non-sustained ventricular tachycardia (VT) on a 24 hour ECG, and inducible VT on electrophysiological testing should have an ICD inserted. That strategy requires extensive investigation of large numbers of patients and the strategy has not yet been tested in clinical trials. The most relevant trials are MADIT and MUSTT in which $52 \%$ and $58 \%$ of patients were on diuretics and the ejection fractions were $26 \%$ and $29 \%$, respectively. In MADIT (multicenter automatic defibrillator implantation trial) $\beta$ blockers were used in ICD patients and controls in 31\% and $14 \%$, respectively. For MUSTT (multicenter unsustained trachycardia trial) the corresponding figures were $29 \%$ and $51 \%$. The low and unbalanced use of $\beta$ blockers makes the interpretation of the trials difficult. MADIT had 196 patients and showed a reduction in mortality of 37\% $(\mathrm{p}<0.01)$.
MUSTT had 704 patients and showed a trend to a reduction in all cause mortality $(p<0.06)$ and a reduction in cardiac arrest or death from arrhythmia $(\mathrm{p}<0.04)$. In the CABG Patch trial (900 patients), in which $50 \%$ of patients had heart failure and the mean ejection fraction was $27 \%$, there was no benefit from ICDs. There are currently a number of trials assessing the value of ICDs in the context of heart failure (MADIT 2, DEFINITE and SCD-HeFT) or after myocardial infarction (DYNAMIT).

The implications of the recommendations from NICE might be considerable. Non-sustained VT is common in patients with heart failure and enlarged hearts; many of these patients are likely to have inducible VT depending on the degree of aggression in the chosen investigative protocol. It would seem unreasonable to be making unguarded recommendations when current trials are conflicting and so many trials are still in progress. The implication is that NICE believes the answer is already known in which case the trials would be unethical. The American guidelines from 1998 (before the publication of MUSTT) gave a grading of class II B (conflicting evidence, divergence of opinion, data derived from a limited number of trials) to the indication as written (section 1.1.2). More careful wording from NICE would have been appropriate.

\section{TECHNICAL APPRAISAL GUIDANCE 12: GUIDANCE ON THE USE OF GLYCOPROTEIN IIB/IIIA INHIBITORS IN THE TREATMENT OF ACUTE CORONARY SYNDROMES}

This document is perhaps the most surprising of the three published so far. The guidance suggests that "For high risk patients with unstable angina or non-Q wave myocardial infarction the intravenous use of the glycoprotein IIb/IIIa inhibitors ... is recommended", and "For patients undergoing acute or elective percutaneous coronary intervention (PCI), the intravenous use of GP IIb/IIIa inhibitors (consistent with current licensing) is recommended". Neither of these recommendations represents current practice in the UK and the cost would be substantial were this advice to be followed.

The recommendations conflict with those in the British National Formulary (No 40, September 2000) for this group of drugs. For abciximab the formulary states as one indication: "Short-term prevention of myocardial infarction in patients with unstable angina not responding to conventional treatment and who are scheduled for percutaneous coronary intervention (use under specialist supervision)". For eptifibatide the formulary states: "Prevention of early myocardial infarction in patients with unstable angina or non-Q wave myocardial infarction and last episode of chest pain within 24 hours (use under specialist supervision)". For tirofiban the wording is similar apart from the requirement of chest pain within 12 hours. NICE has become entangled in an ongoing debate confused by more recent trial data and complicated by differences within trials in the rates of intervention, the nature of the coronary lesion, and the indication for intervention. Current recommendations from USA and Europe are also influenced by the different rates of access to interventional procedures.

The authors of this document had sight of ESPRIT (European study of prevention of reocclusion after initial thrombolysis) (not yet published and assessed by the profession but some data available on the web), but possibly not of other recently reported but unpublished studies; ESPRIT may have had an undue influence on their opinions. ESPRIT was concerned with testing eptifibatide in the context of the insertion of stents. Only $38.5 \%$ of patients had an admission diagnosis of stable angina and therefore could be considered eligible for elective procedures. The 30 day outcome for death or major myocardial infarction was $5.5 \%$ versus $3.7 \%$ in the treated group. These figures are far too high for the procedures to be regarded as elective. 
The GUSTO-IV ACS trial with abciximab for acute coronary syndromes showed that in the absence of early intervention, treatment did not reduce the incidence of death or myocardial infarction and increased the rate of bleeding. Earlier trials had suggested modest benefits in the form of a definite reduction in death or myocardial infarction early after the acute event. A limitation of the data is the short follow up and the fact that the end points are often determined not by death but by myocardial infarction which may include small infarcts, possibly of little long term prognostic significance. Other recent data on oral rather than intravenous administration, but nevertheless indicating potential harm, is not identified. Oral agents were used in OPUS-TIMI 16, EXCITE, SYMPHONY, and BRAVO; none of these trials indicated a benefit and some showed harm.

The most surprising suggestion in this guidance is that all patients undergoing percutaneous coronary intervention including elective cases should receive these drugs.

That is far from current practice where such drugs are reserved for patients with complicated lesions. The trials, presumably used to support this claim, contain an unknown number of patients with complex lesions; the clinical indications for intervention vary. The recent CADILLAC trial of abciximab in the context of myocardial infarction showed no benefit regardless of whether a stent was inserted or simple percutaneous intervention was undertaken without the insertion of a stent. The TARGET trial claimed to show superiority of abciximab compared to tirofiban in patients undergoing coronary stenting. None of these trials, including the ESPRIT trial, which may have influenced these recommendations from NICE, have yet been published in peer reviewed journals and thus subjected to critical examination by the profession. Unresolved issues are oral versus intravenous preparations, class effect or drug differences, dose, timing of drug administration, duration of action, clinical indication, validity of end points in trials, and access to intervention. The appraisal minimises the complexities of the clinical situations which face physicians and largely ignores many pharmacological issues relating to this class of drug.

\section{DISCUSSION}

NICE must be judged by the standards it has set itself. Other organisations such as the American College of Cardiology, the American Heart Association, the European Society of Cardiology, national societies, and health groups have issued guidelines on some or all of these topics. The criticisms propounded by NICE of guidelines from elsewhere are not original and well known. Other bodies have taken considerable care to circumvent these problems and have often chosen to indicate the strength of their recommendations and of the quality of the evidence on which they are based. NICE has not done that nor has it always consulted widely or appropriately in issuing guidance. The recommendations are remarkable for the extent to which they diverge from current practice. There is little evidence that bias, arbitrary decision making, and opinion have been avoided.

The Appraisal Committee has apparently had access to some unpublished trials but not others; the committee has fallen into the trap of bias in the selection of trials. In any event, basing guidelines on unpublished data is not acceptable. The medical community has not had the opportunity to subject these findings to scrutiny.
The most serious charge against the current appraisals is that the authors have been guessing. Time may show that the use of stents should replace surgery in the management of angina, that the wider use of ICDs is advantageous, and that IIb/IIIa inhibitors do have an important role in cardiovascular medicine. But it is most inappropriate for guidance under the aegis of authority to be based on the prejudices of the authors and premature judgements. Indeed a degree of conservatism in medicine may avoid the harm of the enthusiast. The desire to be topical and relevant to current controversy seems to have overwhelmed the need for careful assessment in making statements which are expected to have wide application. Where there is reasonable doubt and uncertainty, that doubt should be explicit so that both the public and the medical profession are informed of the limits to medical knowledge.

Many physicians initially viewed NICE as an attempt by the government to limit expenditure. These recommendations appear to contradict that view since the recommendations will have substantial financial implications for any hospital trust. The net additional costs to the National Health Service are estimated to be greater than $£ 15$ million for IIb/IIIa inhibitors alone. The additional cost of ICDs is put at $£ 30$ million, excluding the costs of the many investigations needed to identify those who should receive such treatment. The cost of applying the policy on stents is not estimated. The overall costs associated with these three documents amounts to substantially more than $£ 50$ million. There is no indication as to whether new funding will be available or what should not be undertaken in order to make savings sufficient to cover these costs.

Physicians will also be concerned about the legal status of guidance from NICE and the dilemma created by conflicts with other published documents such as the British National Formulary. That concern is partly mitigated by the requirement for the physician to exercise clinical judgement. But then the guidelines lose most of their authority to bring about and implement change; the guidelines become in effect merely yet another guideline or consensus document. If the guidance from NICE is not a guideline but a direction to follow in the murky area of current controversy, then that should be made explicit. The guidance becomes a contribution to debate not a statement with authority.

Heart disease is and will remain for the next few decades the most important single health problem and cause of death in the UK. NICE was an important initiative for cardiovascular medicine in that it had the authority to promote the proper and widespread use of established treatments. In many areas such as prevention of coronary heart disease and treatment of heart failure known effective treatments are not being fully implemented. Instead NICE has reported on a curious selection of topics not central to the management of patients. Future documents might usefully provide guidance on prevention of coronary disease, the treatment of heart failure, the use and timing of different types of intervention in the management of angina, the treatment of atrial fibrillation, and the management of acute coronary syndromes and myocardial infarction. When NICE gets round to issuing guidance on these major topics, it should learn from the mistakes of the past. Physicians deserve better. Clinical matters are complicated and those complexities cannot be ignored and subsumed in simplistic and potentially misleading pronouncements. Authority is earned by respect. 